\title{
Preliminary status of POLICAN: A near-infrared imaging polarimeter
}

\author{
R. Devaraj, A. Luna, L. Carrasco and Y. D. Mayya \\ Instituto Nacional de Astrofísica, Óptica y Electrónica \\ Puebla - 72840, México \\ email: dev@inaoep.mx, aluna@inaoep.mx, carrasco@inaoep.mx and ydm@inaoep.mx
}

\begin{abstract}
POLICAN is a near-infrared $(\mathrm{J}, \mathrm{H}, \mathrm{K})$ imaging polarimeter developed for the Cananea near infrared camera (CANICA) at the $2.1 \mathrm{~m}$ telescope of the Guillermo Haro Astrophysical Observatory (OAGH) located at Cananea, Sonora, México. The camera has a 1024 x $1024 \mathrm{HgCdTe}$ detector (HAWAII array) with a plate scale of $0.32 \mathrm{arcsec} /$ pixel providing a field of view of $5.5 \times 5.5$ arcmin. POLICAN is mounted externally to CANICA for narrow-field (f/12) linear polarimetric observations. It consists of a rotating super achromatic $(1-2.7 \mu \mathrm{m})$ half waveplate and a fixed wire-grid polarizer as the analyzer. The light is modulated by setting the half waveplate at different angles $\left(0^{\circ}, 22.5^{\circ}, 45^{\circ}, 67.5^{\circ}\right)$ and linear combinations of the Stokes parameters $(I, Q$ and $U)$ are obtained. Image reduction and removal of instrumental polarization consist of dark noise subtraction, polarimetric flat fielding and background sky subtraction. Polarimetric calibration is performed by observing polarization standards available in the literature. The astrometry correction is performed by matching common stars with the Two Micron All Sky Survey. POLICAN's bright and limiting magnitudes are approximately 6 th and 16 th magnitude, which correspond to saturation and photon noise, respectively. POLICAN currently achieves a polarimetric accuracy about $3.0 \%$ and polarization angle uncertainties within $3^{\circ}$. Preliminary observations of star forming regions are being carried out in order to study their magnetic field properties.
\end{abstract}

Keywords. Near-infrared, Half waveplate, Polarization, Position angle, Magnetic fields

\section{Introduction}

The Guillermo Haro Astrophysical Observatory (OAGH) in Cananea, Sonora, México has a $2.1 \mathrm{~m}$ Ritchey-Chrétien reflector telescope which is situated at an altitude of $2480 \mathrm{~m}$. The optical setup provides images with a PSF of 1.5 arcsec FWHM. A near infrared camera called the Cananea near infrared camera (CANICA) is a regular detector at the observatory which has been used for various astronomical studies in the near-infrared (NIR) since 2002. In order to exploit the NIR capabilities of the camera, the polarimeter POLICAN was developed in 2012. At NIR wavelengths, polarimetric observations provide information about magnetic fields of nebulas, AGNs and, particularly, of star forming regions in molecular clouds, where the polarization is due to dust absorption (e.g. Jones, 1989; Nishiyama et al. 2009; Chapman, 2011 ). Existing NIR polarimeters like MIMIR (Clemens, 2007) and SIRPOL (Kandori et al. 2006) which operate at similar telescopes, have been used to conduct various observations and surveys like GPIPS (Clemens et al. 2012) to understand the role of magnetic fields in star forming regions. POLICAN will complement and aid in doing NIR polarimetric studies as a dedicated instrument in the coming years. 


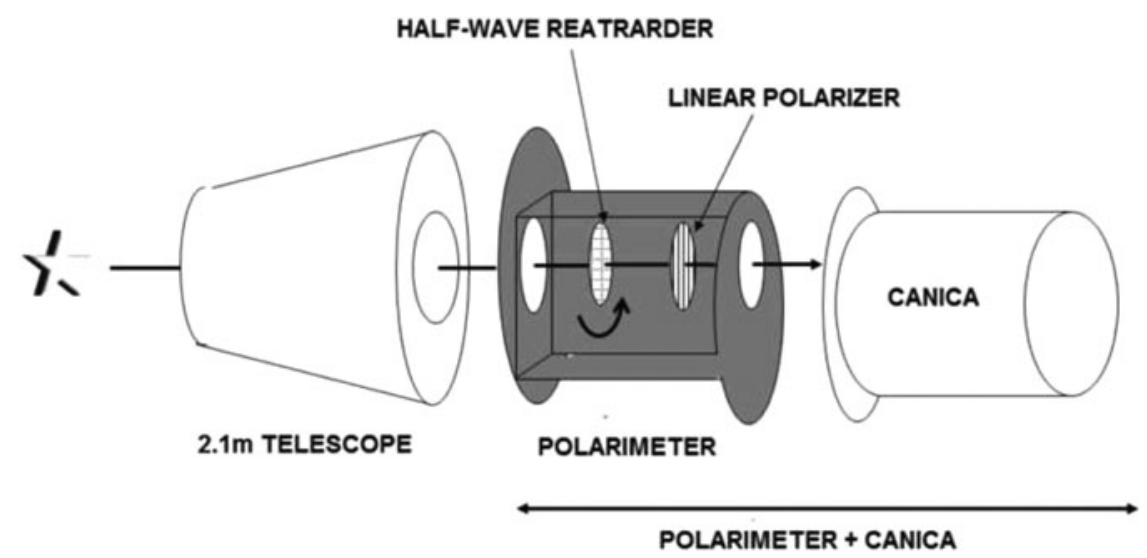

Figure 1. Block diagram of the POLICAN instrument with the telescope and the CANICA detector. The half waveplate with the rotating unit and the polarizer are housed in a mechanical assembly that can be coupled to the telescope on one end and to CANICA on the other.

\section{Design and Hardware}

POLICAN was designed and constructed using principle polarizing elements consisting of a retarder (half waveplate - HWP) and an analyzer (linear polarizer) which are located upstream of the camera at the Cassegrain focus. The Analyzer is a Holographic HER (High extinction ratio) $71 \mathrm{~mm}$ diameter polarizer which is made up of $\mathrm{CaF}_{2}$ and is manufactured by Specac. The retarder is a superachromatic $(1-2.7 \mu \mathrm{m})$ half waveplate of diameter $50 \mathrm{~mm}$ which is made of $\mathrm{MgF}_{2}$ and is manufactured by Bernhard Halle Nachfl. The HWP is fixed to a rotator unit run by a stepper motor, and its function is to modulate at various position angles $\left(0^{\circ}, 22.5^{\circ}, 45^{\circ}, 67.5^{\circ}\right)$. The motor operation is handled by a micro-controller that is operated from the main observation computer. All the elements of the polarimeter are housed inside a mechanical assembly kept at room temperature. This assembly interfaces with the telescope on one end and with CANICA on the other, as shown in Fig. 1. The optical elements of the polarimeter are detachable, thus enabling switching between the normal photometry and the imaging polarimetry modes. The deflection in the mechanical assembly due to the weight of CANICA at different declinations was found to be negligible.

\section{Detector characteristics and performance}

The CANICA camera is based on the 1024 x 1024 Rockwell Science Center HAWAII (HgCdTe Astronomical Wide Area Infrared Imaging) focal plane array (FPA) with mean Quantum efficinecy (QE) of 76\%. It includes a cryostat with two 15-position filter wheels and a pupil mask. The cryostat contains the single fixed re-imaging optics, two 15position filter wheels, the corresponding drive motors and position encoders, the FPA, temperature sensors, and electrical connections. The operating temperature is $77 \mathrm{~K}$. The optics accept a f/12 beam over an unvignetted field of 4.0 × 4.0 arcmin for direct imaging at the Cassegrain focus. The fixed optical design reimages the input beam at $\mathrm{f} / 6$ on the FPA, providing a magnification ratio of $2: 1$. This yields a 0.32 arcsecond/pixel scale. The integrated transmission of the CANICA camera optics (without the filters) is designed to be greater than $85 \%$ for all wavelengths in the 0.85 to $2.4 \mu \mathrm{m}$ spectral range.

The photometric performance of the detector for bright and faint limits were the 6 th and the 16 th mag, set by saturation and photon noise, respectively. The limiting 
magnitudes were estimated to be $\mathrm{J}=17.5, \mathrm{H}=17.2$ and $\mathrm{K}=16.0$ (at $\mathrm{S} / \mathrm{N}=10$ ) with zero points of $\mathrm{J}=20.40, \mathrm{H}=20.50$ and $\mathrm{K}=20.10$. Currently, the intrinsic photometric error of the instrument is found to be around 0.05 magnitudes, at best, which corresponds to $3.0 \%$ in the polarization accuracy.

\section{Observing scheme}

POLICAN saw its first light in November 2013. The observation scheme for POLICAN is being optimized to obtain high $\mathrm{S} / \mathrm{N}$ images in order to reach good polarimetric accuracy. Observations are carried out by acquiring a number of dithered images for each of the four HWP position angles $\left(0^{\circ}, 22.5^{\circ}, 45^{\circ}, 67.5^{\circ}\right)$. For extended sources, an observation set consists of 12 images with alternating source and off-field sky. For point sources, an observation set consists of images obtained at seven dither positions, distributed over a hex-pattern with a diameter of 20 arcsec. Image acquisition is carried out with help of a user interface driven by a second generation San Diego State University (SDSU) CCD controller. The automatic observing sequence is passed to the telescope through a Java script for which the coordinates have to be entered manually. The modulation of the polarimeter for different HWP position angles is controlled by a separate user interface. Dark frames are obtained by taking multiple darks at the end of the observing night for all the exposure times. The polarimetric flat fields are obtained by pointing at the dome's flat-field screen. The flats consists of lights-On and lights-Off sets taken at each of the $4 \mathrm{HWP}$ position angles. Each of these sets is repeated 30 times, for a total of 60 On-Off image pairs per HWP position angle to ensure high S/N. Exposure times are set to half-fill the detector's pixel wells. The On-Off pairs are subtracted in order to remove the effects of dark noise and thermal emission. Then they are averaged for each HWP position angle. On a typical night POLICAN produces around 500 images which approximately sum up to $1 \mathrm{~GB}$ of memory.

\section{Image reduction and analysis}

The challenges to obtaining high-quality linear imaging polarimetric data from the POLICAN instrument include (1) the pixel dark current, which is not linear with exposure time, (2) time-dependent variations of the atmospheric background and sky transmission, (3) a large number of bad pixels spread across the array, (4) the pixel to pixel variations, with a different responses for each exposure time. To obtain measures of the linear polarization and position angles (P.A.) of stars in each observation, two distinct stages of data processing are needed. The first stage consists of the normal steps of basic image correction leading to science quality images. The second stage performes multiple analyses and corrections to yield polarization percentages, P.A.s, and their uncertainties. Stage 1: The first stage of data processing leading to science quality images uses a custom based IRAF pipeline. Processing consists of associating all the images of one particular set of observations with their corresponding averaged dark frames and averaged polarimetric flats. The pipeline removes the dark noise contribution and computes the median sky value from all the dithered images. Then it uses it to remove the sky contribution and the bad pixels. The pixel to pixel variation and any effects introduced by the HWP are corrected using polarimetric flats, which are normalized to the median-filtered mean value for a large sub-sample of pixels chosen to span the central region of the camera. The next step involves selecting and saving common star coordinates in each dithered frame for final combination. From the computed image shifts of selected stars, the pipeline aligns the dithered frames and then averages them to produce one final master frame. 
These steps are repeated for each of the 4 different HWP position angles to obtain their corresponding final master frames. In the end the pipeline does photometry for all the stars detected in the final 4 master frames and outputs a text file which contains Xcenter, Ycenter, Flux, Magnitude and Magnitude error values. The images are Astrometry corrected by matching them to the common stars in the Two Micron All Sky Survey (Skrutskie et al. 2006).

Stage 2: The output file from Stage 1 consists of star coordinates and their corresponding photometric information. These are then processed in a new custom based program developed with IDL software. The program reads the output file for the final 4 master frames and converts the zero point corrected magnitudes and errors to their corresponding fluxes and flux errors. The coordinates for each star in an image are then matched (within a several pixel radius) with the coordinates of the stars in the other images, prior to giving them an identification number. The Stokes $Q$ and $U$ in the instrumental reference system are computed from the fluxes $I_{0}, I_{22.5}, I_{45}, I_{67.5}$ as follows:

$$
\begin{gathered}
Q=\left(I_{0}-I_{45}\right) /\left(I_{0}+I_{45}\right) \\
U=\left(I_{22.5}-I_{67.5}\right) /\left(I_{22.5}+I_{67.5}\right)
\end{gathered}
$$

These are scaled by the polarization efficiency, $\eta$, and rotated by the HWP zero-phaseoffset angle, $\theta$, of the instrument's coordinate system relative to the sky's equatorial coordinate system:

$$
\begin{aligned}
& Q_{e q}=(Q \cos (2 \theta)-U \sin (2 \theta)) / \eta \\
& U_{e q}=(U \cos (2 \theta)+Q \sin (2 \theta)) / \eta
\end{aligned}
$$

Finally, they are combined to form the equatorial polarization percentage $P_{e q}$ and the $P . A$ (measured eastwards from the North) along with the polarization uncertainty $\sigma P$ computed from the corresponding Stokes errors.

$$
\begin{gathered}
P_{e q}=100 \sqrt{Q_{e q}^{2}+U_{e q}^{2}} \\
P . A=\frac{1}{2} \tan ^{-1}\left(\frac{U_{e q}}{Q_{e q}}\right) .
\end{gathered}
$$

The Ricean correction prescription of Wardle \& Kronberg (1974) is used to de-bias the percentage polarization:

$$
P=\sqrt{P_{e q}^{2}-\sigma P^{2}}
$$

The P.A. uncertainity was computed as:

$$
\sigma P . A=28.65\left(\frac{\sigma P}{P}\right)
$$

\section{Polarimetric Calibration}

The polarimetric calibration required the determination of the zero-phase offset angle, $\theta$, of POLICAN's HWP relative to the equatorial coordinate system. This was achieved by observing the polarimetric standards listed in Whittet et al. (1992) and MIMIR (Clemens, 2012). Multiple observations of standard stars HD38563C and CygnusOB221, in the fields of Orion and Cygnus, were carried out for number of nights. These observations showed polarization values consistent with the published ones. The correction of the position 
HD38563C $\mathrm{H}$-band polarization \%

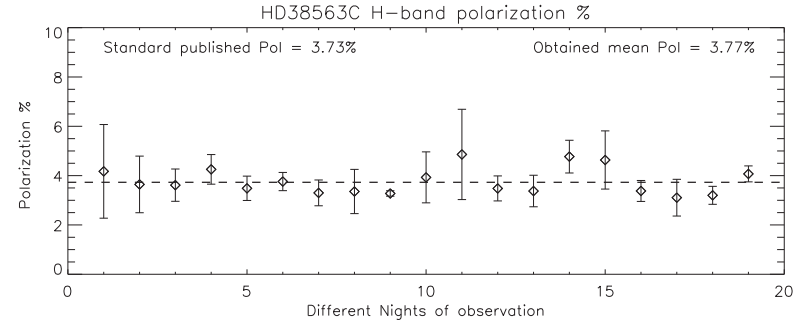

HD38563C $\mathrm{H}$-band polarization PA

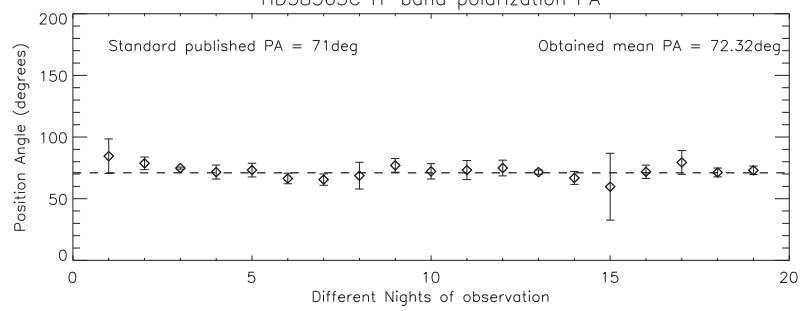

Figure 2. Plot of the calculated polarization and position angle of standard HD38563C over different nights of observation. The dashed line represents the standard published values. The computed mean $P$ and $P$.A for each night are shown by the diamond symbols, along with their corresponding error bars.

angle to translate POLICAN's measurements to the equatorial coordinate system was estimated to be $139^{\circ}$. Results for HD38563C corrected for position angle are shown in Fig. 2. Observations of globular clusters are being carried out to determine the remaining instrumental polarization for final calibration.

\section{Observation}

In Fig. 3 we show SIRPOL and POLICAN observations of Sh2106 in the H-band. This is an emission nebula and an H II region (Sharpless, 1959) in the Cygnus constellation, estimated to be at a distance of 600 pc. At the center of the nebula is a young massive star (type O8) of approximately 15 solar masses that emits jets of gas, forming a bipolar structure. Dust surrounding the star is also illuminated by the central star. Observations of the region around the central massive star carried out with POLICAN showed high polarization levels, which appear in a centro-symmetric pattern, as had been previously shown by Saito et al. (2009). This indicates that the nebulosity is associated to the central star, and that it is responsible for the structure of the circumstellar matter (e.g., disk/envelope system), which is producing the strongly polarized light via dust scattering. Aperture polarimetry for measuring the integrated polarization of background stars was performed to provide information on the direction of the magnetic fields.

\section{Summary}

We have briefly described the design, performance, calibration and initial results of the polarimeter POLICAN developed for the near-infrared camera CANICA installed at the OAGH $2.1 \mathrm{~m}$ telescope, in Mexico. The combination of the polarimeter with CANICA enables deep narrow-field (5.5 x 5.5 arcmin) imaging polarimetry in the JHK bands. The calibration of POLICAN is being optimized to operate at the instrument limits and to meet our desired specifications. Various observations of star forming regions in 

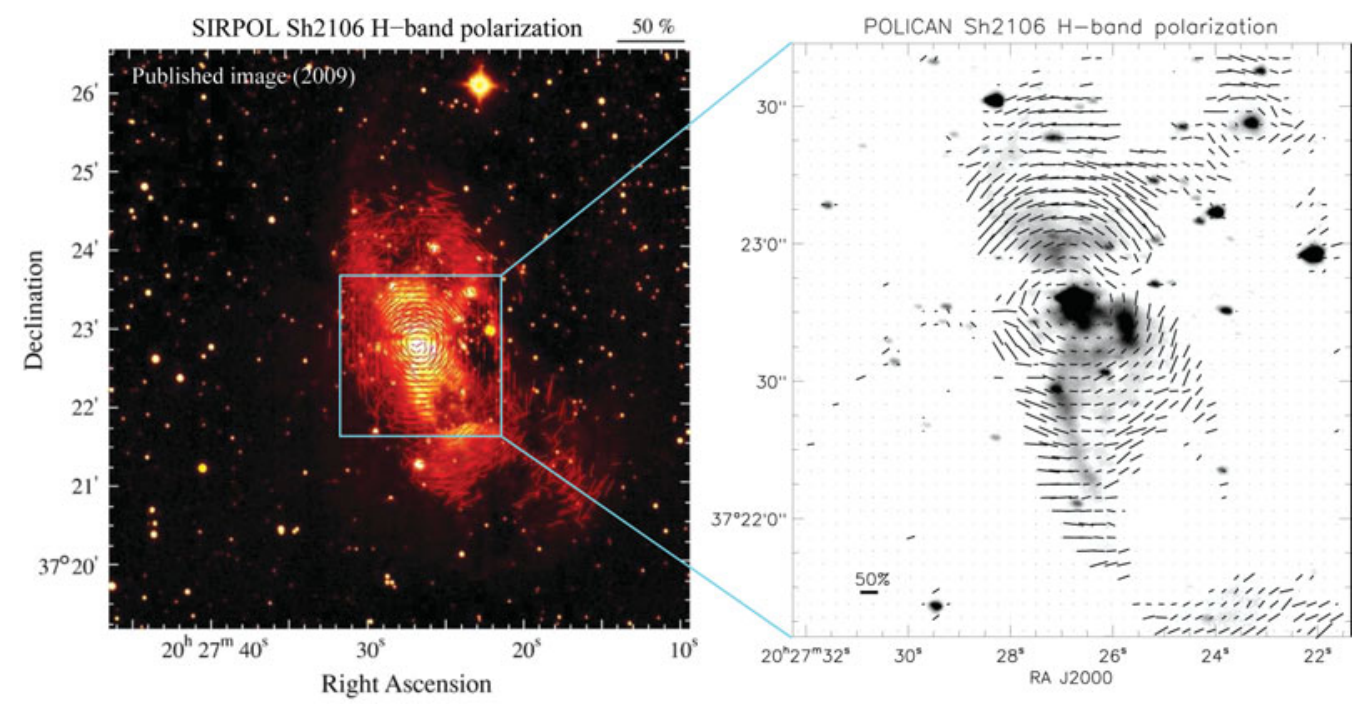

Figure 3. (Left) SIRPOL H-band observation of Sh2106 published in Saito et al. (2009). (Right) POLICAN's H-band observation of Sh2106 central region showing polarization map overlaid on total intensity image. The vectors are plotted for levels above $5 \sigma$ with binning size of $8 \times 8$ pixels. The map reveals a centro-symmetric polarization pattern around the central massive star.

the galactic plane are being conducted to study their magnetic field properties from the polarization data.

\section{Acknowledgements}

We thank all the OAGH staff for their help in the development and the observations with the instrument. This work has been carried out at the Instituto Nacional de Astrofísica, Óptica y Electrónica, México with support from CONACYT under the project CB-2012-01 182841. D.R. with CVU 555629 acknowledges CONACYT for the PhD grant.

\section{References}

Chapman, N. L., Goldsmith, P. F., Pineda, J. L., \& Clemens, D. P. 2011, ApJ 741, 21

Clemens, D. P., Sarcia, D.,Grabau, A., Tollestrup, E. V., Buie, M. W., Dunham, E., \& Taylor, B. 2007, PASP 119, 1385

Clemens, D. P., Pinnick, A., \& Pavel, M. D. 2012, ApJS 200, 20

Clemens, D. P., Pinnick, A., Pavel, M. D., \& Taylor, B. W. 2012, ApJS 200, 19

Jones, T. J. 1989, ApJ 346, 728

Kandori, R., Kusakabe, N., Tamura, M., Nakajima, Y., Nagayama, T., Nagashima, C., Hashimoto, J., Hough, J., Sato, S., Nagata, T., and 3 coauthors 2006, SIRPOL: a JHKssimultaneous imaging polarimeter for the IRSF 1.4-m telescope, Proc SPIE 6269, 159

Nishiyama, S., Tamura, M., Hatano, H., Kanai, S., Kanai, S., Kurita, M.,; Sato, S., Matsunaga, N., Nagata, N., Nagayama, T,. Kandori, R., and 6 coauthors 2009, ApJ 690, 1648

Saito, H., Tamura, M., Kandori, R., Kusakabe, N., Kusakabe, N., Hashimoto, J., Nakajima, Y., Sato, Y., Nagata, T., Nagayama, T., \& Kato, D., 2009, AJ 137, 3149

Stewart sharpless 1959, ApJS 4, 257S

Skrutskie, M. F., Cutri, R. M., Stiening, R., Weinberg, M. D., Schneider, S., Carpenter, J. M., Beichman, C., Capps, R., Chester, T., Elias, J., and 21 coauthors 2006, AJ 131, 1163

Wardle, J. F. C. \& Kronberg, P. P. 1974, ApJ 194, 249

Whittet, D. C. B., Martin, P. G., Hough, J. H., Rouse, M. F., Bailey, J. A., \& Axon, D. J. 1992, ApJ 386, 562 